Transcontinentales

continentales

Sociétés, idéologies, système mondial

$4 \mid 2007$

Amérique latine

\title{
La démocratie locale en Inde
}

Girish KUMAR, Local Democracy in India. Interpreting Decentralization, New Delhi, Sage Publications, 2006, 326 p.

Loraine Kennedy

\section{OpenEdition}

Journals

Édition électronique

URL : http://journals.openedition.org/transcontinentales/1011

DOI : $10.4000 /$ transcontinentales. 1011

ISBN : 978-2-7351-1565-5

ISSN : $1775-397 X$

Éditeur

Editions de la maison des sciences de l'homme

Édition imprimée

Date de publication : 1 juin 2007

Pagination : 173-175

ISBN : 978-2-200-92396-9

ISSN : 1950-1684

Référence électronique

Loraine Kennedy, « La démocratie locale en Inde », Transcontinentales [En ligne], 4 | 2007, document

12, mis en ligne le 04 mai 2011, consulté le 25 septembre 2020. URL : http://journals.openedition.org/ transcontinentales/1011 ; DOI : https://doi.org/10.4000/transcontinentales.1011 
les équipes sanitaires. Renvoyer à un tel exemple de dysfonctionnement ne relève pas de l'anecdote, mais incite à ne pas se contenter de mettre en avant des bribes de projets qui marchent et de passer sous silence d'autres actions, maladroites et incomplètes, qui en toute apparence ne répondent pas aux attentes des personnes victimes du VIH.

C'est d'ailleurs ce que tend à exprimer ce livre: trop de connaissances relatives aux contraintes et aux vécus des populations (soignants et soignés) restent encore ignorées, alors qu'il est désormais prouvé qu'une meilleure prise en compte de ces données socioculturelles serait la condition sine qua non pour une amélioration durable des programmes de lutte contre le VIH.

Frédéric Bourdier Institut de recherche pour le développement (IRD)

\section{La démocratie locale en Inde}

\section{Girish KUMAR,}

Local Democracy in India. Interpreting Decentralization,

New Delhi, Sage Publications, 2006, 326 p.

Cet ouvrage d'un chercheur du Centre de sciences humaines de New Delhi propose une lecture critique de l'expérience indienne de décentralisation, à travers les institutions de gouvernement local, dites "panchayati raj». Le terme panchayat renvoie au conseil villageois traditionnel composé de cinq (panch) membres, institution historique réifiée pendant la période coloniale et érigée en idéal par les avocats de l'autogestion villageoise (gram swarajya), dont, au premier rang, le Mahatma Gandhi. À la fin des années 1950, sous l'impulsion du gouvernement central, les États fédérés rédigèrent des lois en faveur des institutions politiques locales, avec trois échelons administratifs: le village (gram panchayat), le bloc, et le district (qui correspond grosso modo à un département français). Cependant, à de rares exceptions près, ces institutions ne fonctionnèrent pas et, au-delà des premiers mandats, les élections ne furent plus guère organisées, apparemment dans l'indifférence générale.

Dans l'étude riche que nous livre Girish Kumar, c'est la toute dernière génération des «PRIs» (panchayati raj institutions), née du $73^{e}$ amendement à la Constitution, qui est au centre de l'analyse. Ce dernier avatar, et son pendant urbain issu du $74^{e}$ amendement, ont nourri les espoirs des partisans de la décentralisation en apportant, entre autres, des garanties de continuité (élections régulières) et des quotas en faveur des femmes (un tiers des sièges) et des groupes socialement défavorisés issus de basses castes ou de populations autochtones (selon leur pourcentage dans la population locale). Les premières évaluations de cette vaste expérience - l'Inde compte environ 250000 villages - commencent tout juste à paraître, et cet ouvrage contribue incontestablement à alimenter l'état des connaissances sur la question, même si l'analyse repose in fine sur un échantillon relativement restreint. Il restitue les résultats des recherches menées dans huit villages situés dans quatre États : Maharashtra, Bengale occidental, Karnataka, et Madhya Pradesh. La structure de l'étude est la suivante: des entretiens à base de questionnaire ont été réalisés dans deux villages de chaque État auprès de tous les élus, et complétés par des discussions informelles avec une partie des élus et des fonctionnaires. Des enquêtes ont également été effectuées auprès des élus des institutions locales aux niveaux supérieurs : ceux du bloc et du district. 
Enfin, dans chaque village ont été interrogées 150 personnes, sélectionnées à partir d'un échantillonnage représentatif des différentes catégories socioprofessionnelles, pour un total de 1200 dans les quatre États.

L'objectif principal de cette recherche est d'évaluer la capacité des institutions de gouvernement local à promouvoir la démocratie. Girish Kumar rappelle les apports théoriques des PRIs, qui lui servent de critères dans son analyse des réalités indiennes : étendre la base sociale de la démocratie en ouvrant à tous la possibilité de participation politique; rendre le système politique plus responsable, plus réactif aux besoins des citoyens et plus transparent; améliorer les services publics à l'échelle locale, notamment l'éducation et les soins primaires; faciliter l'expression des demandes de la part de la population locale; fournir un forum pour l'échange des points de vue et pour la définition collective des priorités en matière de développement; constituer un lieu d'apprentissage pour la formation des leaders politiques de l'avenir. Face à ces critères et aux ambitions qu'ils incarnent pour la démocratie participative en Inde, la performance des panchayats étudiés est très décevante. Parmi les problèmes principaux, trop nombreux à détailler, certains viennent de ce que les législations des États ne définissent pas assez précisément les fonctions et les compétences attribuées aux collectivités locales, ce qui est aggravé par une autonomie financière parfois très limitée. Kumar consacre un chapitre aux contextes politiques des quatre États, afin de souligner les facteurs qui expliquent l'attitude des dirigeants régionaux à l'égard des PRIs et de fournir des éléments essentiels sur la culture politique des régions étudiées. Cette attention aux systèmes politiques régionaux, qui prend appui sur les travaux antérieurs de l'auteur, est particulièrement pertinente pour comprendre les différences entre les quatre États et constitue une valeur ajoutée par rapport à la plupart des études qui limitent leur investigation à l'échelle locale. Nous apprenons par exemple qu'au Bengale, où les autorités régionales organisent des élections locales depuis 1977, lorsque la coalition menée par le parti communiste-marxiste (CPI-M) est arrivée au pouvoir, les PRIs sont restées tributaires du parti. Leur performance est globalement meilleure que dans les trois autres États, mais les élus locaux sont subordonnés aux cadres du CPI-M, vers qui ils canalisent les demandes locales. La transparence et la responsabilité font défaut, et la promotion de la participation populaire ne semble pas un objectif recherché.

Kumar pointe la mainmise persistante des élites rurales - et de leurs mandataires - sur les institutions politiques et administratives locales, et affirme que les relations traditionnelles de pouvoir se sont maintenues en dépit d'une plus grande représentation sociale parmi les élus. Les enquêtes montrent que ces derniers ignorent souvent les fonctions officielles du conseil et n'ont qu'une idée vague de leurs responsabilités. Cela étant, ils sont perçus par le public comme un agent facilitateur auprès de l'État: beaucoup d'élus ont fait part des sollicitations pour arbitrer des différends, pour obtenir une carte de rationnement donnant accès à des denrées subventionnées ou à un prêt bancaire, ou encore pour bénéficier de tel ou tel programme social, ce qui témoigne d'une certaine mobilisation. Cependant, le clientélisme reste le mode dominant de cette relation; en effet, de manière générale, les villageois interviewés ne font pas le lien entre gouvernement local et responsabilité publique, et les avantages 
dont ils jouissent, qu'ils soient personnels ou collectifs, sont perçus comme le résultat de la largesse des élites. Cette mentalité, que Kumar qualifie de "féodale», constitue sans doute une entrave à la participation politique: les enquêtes ont confirmé que le gram sabha, espèce d'assemblée générale du village instituée dans un grand nombre d'États en vue de favoriser la participation directe, sert surtout de meeting politique où le public vient présenter des pétitions ou des requêtes pour les aides publiques, et où sont distribuées les faveurs du gouvernement.

Même si cette étude ne livre pas de résultats surprenants pour ceux qui suivent la politique en Inde, la profondeur des enquêtes et la finesse de l'analyse apportent des éclaircissements sur les institutions de gouvernement local et contribuent ainsi à une meilleure compréhension des processus sociaux à l'échelle villageoise dans l'Inde d'aujourd'hui.

Loraine Kennedy CNRS, Centre d'études de l'Inde et de l'Asie du Sud (CNRS-EHESS), Paris

\section{L'Inde et I'Europe}

\section{Rajendra K. JAIN}

et Hartmut ELSENHANS (éds), India, Europe and The Changing Dimensions of Security, New Delhi, Radiant Publishers, 2006, 309 p.

\section{Rajendra K. JAIN}

et Hartmut ELSENHANS (éds), India, the European Union and the WTO, New Delhi, Radiant Publishers, 2006, 316 p.

Rajendra K. JAIN, Hartmut ElseNHANS et Amarjit S. NARANG (éds),

The European Union in World Politics, New Delhi, Radiant Publishers, 2006, 324 p.

Professeur à l'université Jawaharlal Nehru de New Delhi, Rajendra Kumar
Jain est un spécialiste des questions européennes. Il a multiplié ces dernières années, avec le soutien de l'Union européenne et de diverses institutions (dont le Centre de sciences humaines de New Delhi) les colloques bilatéraux IndeEurope, sur des thèmes qui portent moins sur les relations entre les deux entités politiques per se, que sur leurs façons d'aborder de grandes questions internationales. Deux des trois ouvrages collectifs qu'il a publiés en 2006 en portent témoignage.

India, Europe and the Changing Dimensions of Security regroupe quatre types d'articles. Le premier offre des mises au point, par des experts particulièrement qualifiés, sur les positions ou les politiques indiennes et européennes. Ainsi Sergio Mascarenhas traite-t-il du rôle de la société civile dans la politique européenne de sécurité (rôle à peu près nul dans la définition précise des politiques de sécurité, mais non négligeable si l'on considère plus largement la société civile comme un "espace d'intervention et de créativité", apte à faire émerger de nouvelles réponses aux problèmes posés... sous réserve que le système institutionnel puisse s'en nourrir). Le chapitre sur le système anti-terroriste français (Jean-Luc Marret) et ceux sur l'Allemagne et la "guerre contre le terrorisme» (Johann-Hinrich Ernst), ou sur l'Inde et le terrorisme en Asie du Sud (Kulbir Krishan), relèvent d'une approche comparable, tout comme celui de C. Raja Mohan, "sécurité et nouveau millénaire: une perspective indienne». Un deuxième type d'articles étudie les politiques d'un des acteurs en direction de l'autre, ou du champ régional concernant l'autre partenaire: ainsi des études sur l'Europe et l'Afghanistan (Mahendra Ved) ou sur l'Europe et la nucléarisation de l'Asie du Sud (J. Fontanel et 